\title{
THE SECOND HOMOLOGY GROUP OF A GROUP; RELATIONS AMONG COMMUTATORS
}

\section{CLAIR MILLER}

We are concerned with the problem of assigning a group theoretic interpretation to the second homology group $H_{2}(G, J)$ of a group $G$, with integer coefficients, $J[1$, p. 486]. We shall define a new group, $H(G)$, called the associated group of $G$, which is, roughly speaking, the group of all relations satisfied by commutators in $G$, taken modulo those relations which are trivially, or universally, satisfied. (The reader is cautioned not to expect that the associated group of an abelian group necessarily vanishes; we do not regard the statement " $x$ and $y$ commute implies $[x, y]=1$ " as a relation.) We then show that $H(G) \approx H_{2}(G, J)$, so that $H_{2}(G, J)$ gives a measure of the extent to which relations among commutators in $G$ fail to be consequences of universal relations.

For a given group $G$, let $\langle G, G\rangle$ be the free group on all pairs $\langle x, y\rangle$, with $x, y \in G$. There is a natural homomorphism of $\langle G, G\rangle$ onto $[G, G]$ which sends $\langle x, y\rangle$ into $[x, y]$. If $w \in\langle G, G\rangle$, we denote its image in $[G, G]$ by $[w]$, and define $Z(G)$ to be the kernel,

$$
Z(G)=\{w \in\langle G, G\rangle \mid[w]=1\} .
$$

Let $B(G)$ be the normal subgroup of $\langle G, G\rangle$ generated by the relations

$$
\begin{aligned}
\langle x, x\rangle & \sim 1, \\
\langle x, y\rangle & \sim\langle y, x\rangle^{-1}, \\
\langle x y, z\rangle & \sim\langle y, z\rangle^{x}\langle x, z\rangle, \\
\langle y, z\rangle^{x} & \sim\langle x,[y, z]\rangle\langle y, z\rangle, .
\end{aligned}
$$

where $x, y$, and $z$ range over $G$ and by definition

$$
\langle y, z\rangle^{x}=\left\langle y^{x}, z^{x}\right\rangle=\left\langle x y x^{-1}, x z x^{-1}\right\rangle .
$$

In other words $B(G)$ is the normal subgroup generated by all $\langle x, x\rangle$, all $\langle x, y\rangle\langle y, x\rangle$, etc. The symbol $\sim$ shall mean congruence in $\langle G, G\rangle$ mod $B(G)$. Evidently $B(G) \subset Z(G)$, and we define the associated group of $G$ to be

$$
H(G)=Z(G) / B(G) .
$$

If $h: G \rightarrow G^{\prime}$ is a homomorphism, we define

Received by the editors December 3, 1951. 


$$
h_{f}:\langle G, G\rangle \rightarrow\left\langle G^{\prime}, G^{\prime}\right\rangle
$$

by $h_{f}\langle x, y\rangle=\langle h(x), h(y)\rangle$. Then $h_{f}$ carries $Z(G)$ into $Z\left(G^{\prime}\right)$ and $B(G)$ into $B\left(G^{\prime}\right)$, inducing a homomorphism

$$
h_{*}: H(G) \rightarrow H\left(G^{\prime}\right)
$$

which satisfies

$$
(h g)_{*}=h_{*} g_{*}, \quad 0_{*}=0, \quad 1_{*}=1,
$$

where 0 is a zero homomorphism, $0(x)=1$, and 1 is an identity homomorphism, $1(x)=x$.

By inverting both sides of (3) and quoting (2) we obtain

$$
\langle x, y z\rangle \sim\langle x, y\rangle\langle x, z\rangle^{y} .
$$

Of the many consequences of the defining relations of $B(G)$ we shall have need for only the following:

$$
\langle x, y\rangle^{\langle a, b\rangle} \sim\langle x, y\rangle^{[a, b]}
$$

where $\langle x, y\rangle\langle a, b\rangle$ is by definition $\langle a, b\rangle\langle x, y\rangle\langle a, b\rangle^{-1}$,

(7) $[\langle x, y\rangle,\langle a, b\rangle] \sim\langle[x, y],[a, b]\rangle$,

(8) $\left\langle b, b^{\prime}\right\rangle\left\langle a_{0}, b_{0}\right\rangle \sim\left\langle\left[b, b^{\prime}\right], a_{0}\right\rangle\left\langle a_{0},\left[b, b^{\prime}\right] b_{0}\right\rangle\left\langle b, b^{\prime}\right\rangle$,

(9) $\left\langle b, b^{\prime}\right\rangle\left\langle b_{0}, a_{0}\right\rangle \sim\left\langle\left[b, b^{\prime}\right] b_{0}, a_{0}\right\rangle\left\langle a_{0},\left[b, b^{\prime}\right]\right\rangle\left\langle b, b^{\prime}\right\rangle$,

(10) $\left\langle b, b^{\prime}\right\rangle\left\langle a, a^{\prime}\right\rangle \sim\left\langle\left[b, b^{\prime}\right],\left[a, a^{\prime}\right]\right\rangle\left\langle a, a^{\prime}\right\rangle\left\langle b, b^{\prime}\right\rangle$,

(11) $\left\langle x^{n}, x^{s}\right\rangle \sim 1$,

$$
n=0, \pm 1, \cdots ; s=0, \pm 1, \cdots \text {. }
$$

We prove (6) by expanding $\langle a x, b y\rangle$ in two ways, using (3) and ( $\left.3^{\prime}\right)$. We have

$$
\begin{aligned}
\langle a x, b y\rangle & \sim\langle a x, b\rangle\langle a x, y\rangle^{b} \\
& \sim\langle x, b\rangle^{a}\langle a, b\rangle\langle x, y\rangle^{b a}\langle a, y\rangle^{b} .
\end{aligned}
$$

Also

$$
\begin{aligned}
\langle a x, b y\rangle & \sim\langle x, b y\rangle^{a}\langle a, b y\rangle \\
& \sim\langle x, b\rangle^{a}\langle x, y\rangle^{a b}\langle a, b\rangle\langle a, y\rangle^{b} .
\end{aligned}
$$

Comparing, we see that

$$
\langle a, b\rangle\langle x, y\rangle^{b a} \sim\langle x, y\rangle^{a b}\langle a, b\rangle,
$$

or

$$
\langle a, b\rangle\langle x, y\rangle^{b a}\langle a, b\rangle^{-1} \sim\langle x, y\rangle^{a b} .
$$

Replacing $x$ and $y$ by $x^{(b a)^{-1}}$ and $y^{(b a)^{-1}}$ gives 


$$
\langle a, b\rangle\langle x, y\rangle\langle a, b\rangle^{-1} \sim\langle x, y\rangle^{b a(b a)^{-1}}=\langle x, y\rangle^{[a, b]} .
$$

Observe that (7) is a consequence of (6), for

$$
\begin{aligned}
{[\langle x, y\rangle,\langle a, b\rangle] } & =\langle x, y\rangle^{\langle a, b}\langle x, y\rangle^{-1} \\
& \sim\langle x, y\rangle^{[a, b]}\langle x, y\rangle^{-1} \\
& \sim\langle[a, b],[x, y]\rangle\langle x, y\rangle\langle x, y\rangle^{-1} \quad \text { by (4). }
\end{aligned}
$$

Relation (8) is verified by expanding $\left\langle a_{0},\left[b, b^{\prime}\right] b_{0}\right\rangle$ by $\left(3^{\prime}\right)$, giving

$$
\begin{aligned}
\left\langle a_{0},\left[b, b^{\prime}\right] b_{0}\right\rangle & \sim\left\langle a_{0},\left[b, b^{\prime}\right]\right\rangle\left\langle a_{0}, b_{0}\right\rangle^{\left[b, b^{\prime}\right]} \\
& \sim\left\langle a_{0},\left[b, b^{\prime}\right]\right\rangle\left\langle a_{0}, b_{0}\right\rangle^{\left\langle b, b^{\prime}\right\rangle} \quad \text { by (6). }
\end{aligned}
$$

Substitution in the right member of (8) gives the desired result. Relation (9) is proved similarly. Relation (10) is a restatement of (7). Relation (11) is proved, for non-negative $n$ and $s$, by an induction on $n+s$, using (3) and ( $\left.3^{\prime}\right)$. When $n+s=1$, say $n=0$ and $s=1$, setting $x=z$ and $y=1$ in (3) gives the result. The case of general $n$ and $s$ follows trivially from the non-negative case by using (3).

THEOREM 1. The associated group of a free group is a one-element group.

The case of a free group with an infinite number of generators follows from the case of a free group with a finite number of generators; for if $F$ is free with infinitely many generators and $u \in H(F)$, then $u \in i_{*} H\left(F^{\prime}\right)$, where $F^{\prime}$ is a subgroup of $F$ on finitely many generators, and $i$ is the inclusion. In case the free group $F$ has but one generator, then $H(F)=1$ by virtue of rule (11), $\left\langle x^{n}, x^{s}\right\rangle \sim 1$. The general case of a free group with finitely many generators follows at once by induction from the following

LEммA. If $G=A * B$ is the free product of $A$ and $B$, then $H(G)$ $\approx H(A) \times H(B)$.

Let $i: A \rightarrow G$ and $j: B \rightarrow G$ be the natural injections, and let $p: G \rightarrow A$ and $q: G \rightarrow B$ be the natural projections. Inspection of the diagram

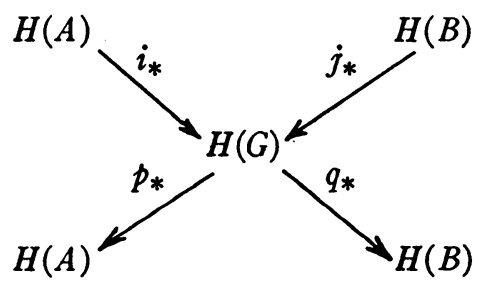

shows that $i_{*}$ and $j_{*}$ are isomorphisms into and that $i_{*} H(A)$ and 
$j_{*} H(B)$ are disjoint. In case $H(G)$ is the group product $i_{*} H(A) j_{*} H(B)$ the diagram also shows that $H(G)$ is the direct product $H(G)$ $=i_{*} H(A) \times j_{*} H(B)$. The problem, then, is to demonstrate that $H(G)$ $=i_{*} H(A) j_{*} H(B)$.

In order to do this we shall be concerned with three subgroups of $\langle G, G\rangle: A=i_{\#}\langle A, A\rangle, \mathcal{B}=j_{\sharp}\langle B, B\rangle$, and $\mathcal{X}$, the subgroup of $\langle G, G\rangle$ generated by all elements of the form $\langle a, b\rangle$, with $a \neq 1 \in A$, and $b \neq 1 \in B$. Let $\langle x, y\rangle$ be a generator of $\langle G, G\rangle$, with $x=a_{1} b_{1} \cdots a_{s} b_{s}$, $y=\bar{a}_{1} b_{1} \cdots \bar{a}_{r} b_{r}$, and with $a_{i}, \bar{a}_{j} \in A, b_{i}, \bar{b}_{j} \in B$. By a repeated application of the product rules (3) and $\left(3^{\prime}\right)$ we see that $\langle x, y\rangle$ is congruent $\bmod B(G)$ to a product of elements of the form $\left\langle a, a^{\prime}\right\rangle^{z},\left\langle b, b^{\prime}\right\rangle^{z}$, $\langle a, b\rangle^{z}$, and $\langle b, a\rangle^{z}$, with $a, a^{\prime} \in A, b, b^{\prime} \in B$, and $z \in G$. Each element of this form can in turn be broken down into a product of terms of the same type, without the exponent $z$ appearing, by repeated use of the rules

$$
\begin{aligned}
\left\langle a, a^{\prime}\right\rangle^{a_{0}} & =\left\langle a^{a_{0}}, a^{\prime a_{0}}\right\rangle, \\
\left\langle a, a^{\prime}\right\rangle^{b_{0}} & \sim\left\langle b_{0},\left[a, a^{\prime}\right]\right\rangle\left\langle a, a^{\prime}\right\rangle, \\
\langle a, b\rangle^{a_{0}} & \sim\left\langle a_{0} a, b\right\rangle\left\langle b, a_{0}\right\rangle, \\
\langle a, b\rangle^{b_{0}} & \sim\left\langle b_{0}, a\right\rangle\left\langle a, b_{0} b\right\rangle,
\end{aligned}
$$

and four more similar rules, obtained from these by interchanging $a$ with $b, a_{0}$ with $b_{0}$, and $a^{\prime}$ with $b^{\prime} .\left(5^{\prime}\right)$ and $\left(4^{\prime}\right)$ are restatements of (5) and (4), and (12) and (13) are restatements of (3) and ( $\left.3^{\prime}\right)$, using rule (2), $\langle c, d\rangle^{-1} \sim\langle d, c\rangle$. Thus we see that $\langle x, y\rangle$, and hence any element $w \in\langle G, G\rangle$, is congruent to a product $\pi$ of terms $\left\langle a, a^{\prime}\right\rangle,\left\langle b, b^{\prime}\right\rangle$, $\langle a, b\rangle$, and $\langle b, a\rangle$.

Now take each term $\left\langle b, b^{\prime}\right\rangle$ in $\pi$ and "commute" it to the right (beginning with the farthermost right one and proceeding one at a time) via (8), (9), and (10). Thus we obtain, for the arbitrary element $w$ of $\langle G, G\rangle, w \sim \pi \sim \pi^{\prime} \beta$, with $\beta$ a product of terms $\left\langle b, b^{\prime}\right\rangle$, and $\pi^{\prime}$ a product of terms $\left\langle a, a^{\prime}\right\rangle,\langle a, b\rangle$, and $\langle b, a\rangle$. Now take each term of the form $\left\langle a, a^{\prime}\right\rangle$ in $\pi^{\prime}$ and commute it to the left via the rules dual to (8) and (9) (obtained from them by inversion and interchanging $a$ and $b$ ); this gives

$$
w \sim \pi^{\prime} \beta \sim \alpha \pi^{\prime \prime} \beta,
$$

with $\pi^{\prime \prime}$ involving only terms $\langle a, b\rangle$ and $\langle b, a\rangle$, and $\alpha$ a product of terms $\left\langle a, a^{\prime}\right\rangle$. By replacing each $\langle b, a\rangle$ in $\pi^{\prime \prime}$ by $\langle a, b\rangle^{-1}$ we replace $\pi^{\prime \prime}$ by $\mu \in \mathcal{X}$ and have

$$
w \sim \alpha \mu \beta
$$


with $\alpha \in \mathcal{A}, \beta \in \mathcal{B}$, and $\mu \in \mathcal{X}$.

Now let $w \in Z(G)$, that is, $[w]=1$. Then $[\alpha][\mu][\beta]=[w]=1$, and projecting into $A$ we see that $[\alpha]=1$; similarly, $[\beta]=1$, so that $[\mu]$ $=1$. However $[\mu]=1$ implies that $\mu=1 \in \mathscr{X} \subset\langle G, G\rangle$. To see this, let $\mu$ be written as a reduced word in the free group $\mathcal{X} ; \mu=\left\langle a_{1}, b_{1}\right\rangle \epsilon_{1} \ldots$ $\left\langle a_{p}, b_{p}\right\rangle \epsilon_{p}$, with $\epsilon_{i}= \pm 1, a_{i} \neq 1 \neq b_{i}$. Then, by induction on $p$, we see that $[\mu]$ can be written as a reduced word in the free product $G=A * B$ in which the last two entries are $b_{p}^{-1} a_{p}^{-1}$ if $\epsilon_{p}=-1$, or $a_{p}^{-1} b_{p}^{-1}$ if $\epsilon_{p}=+1$. In particular $[\mu] \neq 1$ if $\mu$ is not the empty word.

Thus $\mu=1$ gives $w \sim \alpha \mu \beta=\alpha \beta$, with $[\alpha]=1$ and $[\beta]=1$, which shows that $H(G)=i_{*} H(A) j_{*} H(B)$ and proves the lemma.

It is possible to use Theorem 1 to show that any "universal relation" among commutators can be deduced from our defining relations (1) to (4). Briefly, a "universal relation" is an expression of the type we have been considering which is valid in any group. We shall not pursue this.

Digressing for a moment, we remark that in proving that $\langle G, G\rangle$ is the group product, $\bmod B(G)$, of $\mathcal{A}, \mathcal{X}$, and $B$ we used only the fact that $G$ is generated by $A$ and $B$. Applying this to the direct product $G=A \times B$ one sees that $H(G)$ is the group product of $i_{*} H(A)$, $j_{*} H(B)$, and $\tau(A \otimes B)$, where $\tau$ is a homomorphism from the tensor product $A \otimes B$ into $H(G)$ defined by requiring that $\tau(a \otimes b)$ is the image in $H(G)$ of $\langle a, b\rangle$. The fact that $\tau$ is a well defined homomorphism follows from the fact that $H(G) \approx H_{2}(G, J)$ is abelian, the congruences

$$
\begin{aligned}
\left\langle a_{1} a_{2}, b\right\rangle & \sim\left\langle a_{2}, b\right\rangle^{a_{1}}\left\langle a_{1}, b\right\rangle \\
& \sim\left\langle a_{1}, 1\right\rangle\left\langle a_{2}, b\right\rangle\left\langle a_{1}, b\right\rangle \\
& \sim\left\langle a_{2}, b\right\rangle\left\langle a_{1}, b\right\rangle
\end{aligned}
$$

and the dual congruence

$$
\left\langle a, b_{1} b_{2}\right\rangle \sim\left\langle a, b_{1}\right\rangle\left\langle a, b_{2}\right\rangle .
$$

If we let $\sigma: H(G) \rightarrow A \otimes B$ be induced by $\bar{\sigma}:\langle G, G\rangle \rightarrow A \otimes B$, where $\bar{\sigma}\left(\left\langle a_{1} b_{1}, a_{2} b_{2}\right\rangle\right)=a_{1} \otimes b_{2}-a_{2} \otimes b_{1}$, an analysis of the diagram

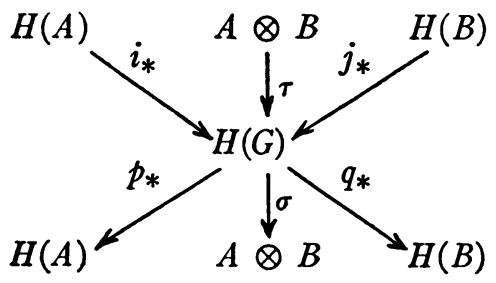


shows that $i_{*}, j_{*}$, and $\tau$ are one-to-one and that

$$
\begin{aligned}
H(G) & =i_{*} H(A) \times j_{*} H(B) \times \tau(A \otimes B) \\
& \approx H(A) \times H(B) \times A \otimes B .
\end{aligned}
$$

THEOREM 2. There is a canonical isomorphism between $H(G)$ and $H_{2}(G, J)$ preserving the notion of induced homomorphism; if $h: G \rightarrow G^{\prime}$ is a homomorphism, we have commutativity in the diagram

$$
\begin{array}{cc}
H(G) & \stackrel{h_{*}}{\longrightarrow} H\left(G^{\prime}\right) \\
\Downarrow & \\
H_{2}(G, J) & \stackrel{h_{*}}{\longrightarrow} H_{2}\left(G^{\prime}, J\right) .
\end{array}
$$

Suppose that $G$ is given as a factor group of a group $E$ by a central subgroup $N$ of $E$. The factoring homomorphism $\eta: E \rightarrow G$ maps $E$ onto $G$ with kernel $N$. We define a homomorphism $\langle G, G\rangle \rightarrow E$ by mapping a generator $\langle x, y\rangle$ of $\langle G, G\rangle$ into $[\bar{x}, \bar{y}]$, where $\eta(\bar{x})=x$ and $\eta(\bar{y})=y$. This is independent of the choices $\bar{x}$ and $\bar{y}$ because $N$ is in the center of $E$. This homomorphism carries $Z(G)$ on to $N \cap[E, E]$ and carries $B(G)$ onto 1 , and hence induces an onto homomorphism $\phi: H(G) \rightarrow N \cap[E, E]$. It is readily verified that the sequence

$$
H(E) \stackrel{\eta_{*}}{\longrightarrow} H(G) \stackrel{\phi}{\longrightarrow} N \cap[E, E]
$$

is exact at $H(G)$, that is, kernel $\phi=$ image $\eta_{*}$.

If $G$ is an arbitrary group, we can represent $G$ as the factor group of a free group $F$ by a subgroup $R, G=F / R$. Letting $F^{0}=F /[F, R]$ and $R^{0}=R /[F, R]$ we have

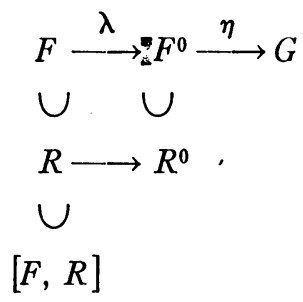

where $\lambda$ and $\eta$ are the factoring homomorphisms. $R^{0}$ is in the center of $F^{0}$, so that $\phi$ maps $H(G)$ onto $R^{0} \cap\left[F^{0}, F^{0}\right]$. By exactness in the sequence $H\left(F^{0}\right) \rightarrow H(G) \rightarrow R^{0} \cap\left[F^{0}, F^{0}\right], \phi$ will be one-to-one provided that $\eta_{*}=0$. To see that this is actually the case, let $w=\left\langle\bar{x}_{1}, y_{1}\right\rangle \ldots$ $\left\langle x_{p}, y_{p}\right\rangle \in Z\left(F^{0}\right)$. Then $[w]=\left[x_{1}, y_{1}\right] \cdots\left[x_{p}, y_{p}\right]=1 \in F^{0}$, and, choosing $\bar{x}_{i}, \bar{y}_{i} \in F$ such that $\lambda\left(\bar{x}_{i}\right)=x_{i}$ and $\lambda\left(\bar{y}_{i}\right)=y_{i}$, we have $\bar{w}=\left\langle x_{1}, \bar{y}_{1}\right\rangle \ldots$ $\left\langle\bar{x}_{p}, \bar{y}_{p}\right\rangle$, with $\lambda_{t} \bar{w}=w, \lambda[\bar{w}]=[w]=1$, and hence $[\bar{w}] \in[F, R]$. 
Therefore $[\bar{w}]=\left[f_{1}, r_{1}\right] \cdots\left[f_{q}, r_{q}\right]$, for some $f_{i} \in F$ and $r_{i} \in R$. However $F$ is free, $H(F)=1$, and $B(F)=Z(F)$. Hence $\bar{w} \sim\left\langle f_{1}, r_{1}\right\rangle \cdots$ $\left\langle f_{\boldsymbol{q}}, \boldsymbol{r}_{\boldsymbol{q}}\right\rangle \bmod B(F)$. Then

$$
\begin{aligned}
\eta_{\sharp} w & =\eta_{t} \lambda_{\sharp} \bar{w} \sim \eta_{\sharp} \lambda_{f}\left(\left\langle f_{1}, r_{1}\right\rangle \cdots\left\langle f_{q}, r_{q}\right\rangle\right) \\
& \sim\left\langle\eta \lambda_{1}, 1\right\rangle \cdots\left\langle\eta \lambda f_{q}, 1\right\rangle \sim 1,
\end{aligned}
$$

and $\eta_{*}=0$.

Thus $\phi: H(G) \approx R^{0} \cap\left[F^{0}, \quad F^{0}\right] . \quad$ However $R^{0} \cap\left[F^{0}, \quad F^{0}\right]=R$ $\cap[F, F] /[F, R]$ is the Hopf construction for $H_{2}(G, J)$, so that we have constructed the desired isomorphism.

A formula can be given for our isomorphism. If $w=\left\langle x_{1}, y_{1}\right\rangle \ldots$ $\left\langle x_{p}, y_{p}\right\rangle \in Z(G)$, then the homology class in $H_{2}(G, J)$ corresponding to the image of $w$ in $H(G)$ is the class of the 2-cycle

$$
\begin{aligned}
\rho(w)= & \sum_{i=1}^{p} g\left(x_{i}, y_{i}\right) \\
& +\sum_{i=1}^{p-1}\left\{\left(\left[x_{1}, y_{1}\right] \cdots\left[x_{i}, y_{i}\right],\left[x_{i+1}, y_{i+1}\right]\right)-(1,1)\right\}
\end{aligned}
$$

where $g(x, y)=(x, y)-(y, x)-\left(y x,(y x)^{-1}\right)+\left(x y,(y x)^{-1}\right)$. When $G$ is abelian this simplifies to

$$
\rho(\langle x, y\rangle)=(x, y)-(y, x) .
$$

A proof of the validity of this formula (we omit it) can be obtained by examining the explicit formulation of the isomorphism $H_{2}(G, J)$ $\approx R^{0} \cap\left[F^{0}, F^{0}\right]$ as given by Eilenberg and MacLane $[1$, p. 485 and 2, p. 75]. (14) shows that the isomorphism is independent of the choice of the representation of $G$ as $F / R$ and that commutativity holds in the diagram as asserted in Theorem 2.

As a simple application of our description of $H_{2}(A, J)$ we give a more detailed analysis of the structure of $H(A)$ (and hence of $H_{2}(A, J)$ also) for an abelian group $A$. Since $Z(A)=\langle A, A\rangle$, and $Z(A) / B(A)=H(A) \approx H_{2}(A, J)$ is abelian, we see that, for the abelian case only, we may as well have taken $\langle A, A\rangle$ to be the free abelian group on the pairs $\langle x, y\rangle$. This we now do. Writing both $A$ and $\langle A, A\rangle$ additively, the defining relations of $B(A)$, together with the consequent relation $\left(3^{\prime}\right)$, become

$$
\begin{aligned}
\langle x, x\rangle & \sim 0, \\
\langle x, y\rangle & \sim-\langle y, x\rangle, \\
\langle x+y, z\rangle & \sim\langle x, z\rangle+\langle y, z\rangle,
\end{aligned}
$$




$$
\begin{aligned}
\langle x, y+z\rangle & \sim\langle x, y\rangle+\langle x, z\rangle, \\
\langle x, 0\rangle & \sim 0 .
\end{aligned}
$$

Observe that $(4 \mathrm{~A})$ is a consequence of $\left(3^{\prime} \mathrm{A}\right)$ by setting $y=0$ in $\left(3^{\prime} \mathrm{A}\right)$. Also, $\langle x+y, x+y\rangle \sim 0$ by (1A), and expanding this by (3A) and $\left(3^{\prime} \mathrm{A}\right)$ shows that $(2 \mathrm{~A})$ is a consequence of $(1 \mathrm{~A}),(3 \mathrm{~A})$, and $\left(3^{\prime} \mathrm{A}\right)$. Thus $B(A)$ can be defined by $(1 \mathrm{~A}),(3 \mathrm{~A})$, and $\left(3^{\prime} \mathrm{A}\right)$, which proves:

Theorem 3. For an abelian group $A, H(A) \approx A \otimes A / D$, where $D$ is the subgroup of the tensor product $A \otimes A$ generated by the diagonal, $\{a \otimes a \mid a \in A\}$.

This gives an isomorphism $A \otimes A / D \approx H_{2}(A, J)$, which, in view of (15), is induced by the homomorphism $A \otimes A \rightarrow H_{2}(A, J)$ in which $x \otimes y$ is mapped into the homology class of $(x, y)-(y, x)$.

\section{REFERENCES}

1. S. Eilenberg and S. MacLane, Relations between homology and homotopy groups of spaces, Ann. of Math. vol. 46 (1945) pp. 480-509. 51-78.

2. - Cohomology theory in abstract groups, I, Ann. of Math. vol. 48 (1947) pp.

3. H. Hopf, Fundamentalgruppe und zweite Bettische Gruppe, Comment. Math. Helv. vol. 14 (1942) pp. 257-309.

University of Chicago 